\title{
Acquired Thrombotic Thrombocytopenic Purpura
}

National Cancer Institute

\section{Source}

National Cancer Institute. Acquired Thrombotic Thrombocytopenic Purpura. NCI

Thesaurus. Code C131653.

Thrombotic thrombocytopenic purpura for which the cause is not present at birth. 